\title{
Hitting the right spot on CXCR4
}

Toll-like receptor 7 (TLR7) and

TLR7-mediated production of type I interferons by plasmacytoid dendritic cells (pDCs) are thought to have central roles in systemic lupus erythematosus (SLE). New findings highlight IT1t, a ligand of the chemokine receptor CXCR4, as an inhibitor of type I interferon production by $\mathrm{pDCs}$, and the IT1t binding site on CXCR4 as a potential therapeutic target in SLE.

Previous studies have shown that a synthetic histamine analogue, clobenpropit, could interact with CXCR4 and inhibit type I interferon production by pDCs. Because clobenpropit also binds histamine receptors, in the new study, the researchers investigated the effects of two CXCR4-specific ligands: AMD3100 (an FDA-approved CXCR4 antagonist) and IT1t (a small amino compound).

The two ligands bind to different sites on CXCR4 and are proposed to have different biological activities.
"Interestingly, IT1t is structurally similar to clobenpropit and was, as expected, the only one of these two ligands that could inhibit type I interferon production by pDCs in vitro," explains corresponding author Jean-Philippe Herbeuval.

In ex vivo tonsil mononuclear cell suspensions, IT1t inhibited TLR7-mediated production of IFNa. Furthermore, IT1t treatment reduced systemic inflammation and autoantibody production, and prevented glomerulonephritis, in a TLR7-dependent mouse model of SLE (pristane-induced lupus).

Treatment with IT1t in vitro also abrogated the production of IFNa by resting and stimulated peripheral blood mononuclear cells from patients with SLE, and normalized the expression of inflammatory gene transcripts in these cells.

"We propose that agonists of CXCR4 could represent a novel class of anti-inflammatory molecules

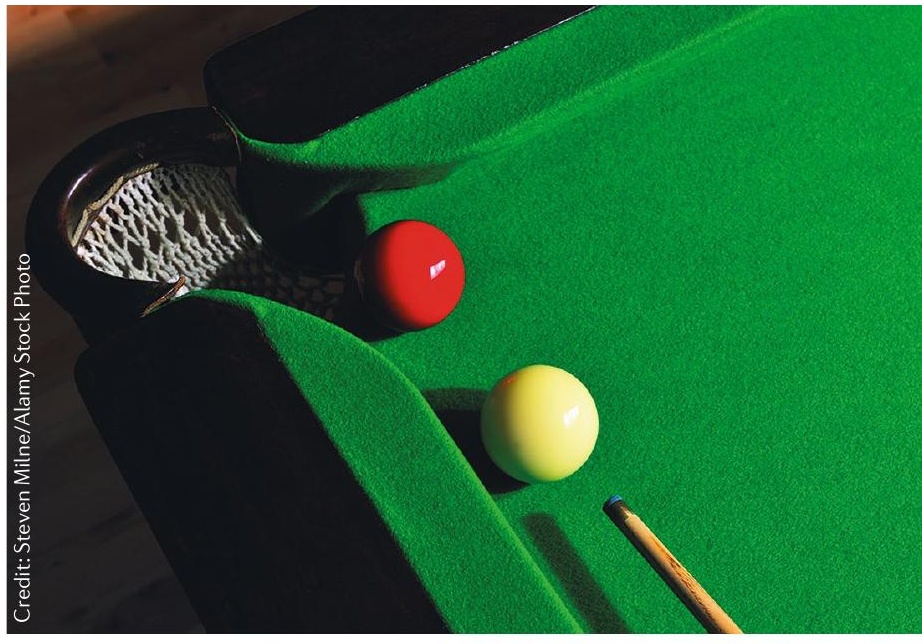

that target CXCR4-positive cells such as pDCs," says Nikaïa Smith, co-corresponding author on the study.

The researchers plan to fully characterize the IT1t binding pocket on CXCR4 and the underlying mechanisms of action, to develop
IT $1 \mathrm{t}$ treatment reduced systemic inflammation and autoantibody
production new CXCR4 ligands that have an even higher efficiency for blocking type I interferon production than IT1t, and to expand these findings to other autoimmune diseases (such as dermatomyositis).

Jessica McHugh

ORIGINAL ARTICLE Smith, N. et al. Control of TLR7-mediated type IIFN signaling in PDCs through CXCR4 engagement - A new target for lupus treatment. Sci. Adv. 5, eaav9019 (2019)

\section{OSTEOARTHRITIS}

\section{Partial knee replacement comes out on TOP(KAT)}

Knee replacement surgery is a common treatment for late-stage osteoarthritis (OA), but evidence as to whether total knee replacement (TKR) or partial knee replacement (PKR) is best has been lacking. TOPKAT, a multicentre randomized controlled trial (RCT), aimed to address questions around the efficacy and cost-effectiveness of TKR versus PKR for knee OA.

“Despite many previous studies and considerable data, we have never had a sufficiently large RCT to answer this important question," says corresponding author David Beard. "TOPKAT has now definitively shown us that both operations provide benefit and are worthwhile but, given the option, PKR is probably the surgery of choice - providing sufficient expertise exists to perform it." In TOPKAT, 528 patients with isolated medial compartment knee OA were recruited from 27 hospitals in the UK. Patients were randomly allocated to receive either TKR or PKR by expert surgeons at a 1:1 ratio and were evaluated for knee pain and function using the Oxford Knee Score (OKS) as the primary outcome at 5 years post-surgery.

"A unique feature of the research was the incorporation of an innovative combined equipoise-expertise based approach to allow surgeons who were not in equipoise (in other words, who had a strong preference for one implant design over another) to participate," explains Beard. "The expertise-based randomization enabled surgeons not in equipoise to participate in the trial by working in pairs, each providing the operation type they felt was appropriate (as 'experts')."

At 5 years follow-up, no difference was seen in the primary outcome between the patients who received PKR and those who received TKR. The incidences of re-operation and adverse events were also similar between the two groups, contrary to previous findings from systematic reviews. Furthermore, PKR was more effective and less expensive than TKR in the cost-effectiveness analysis.

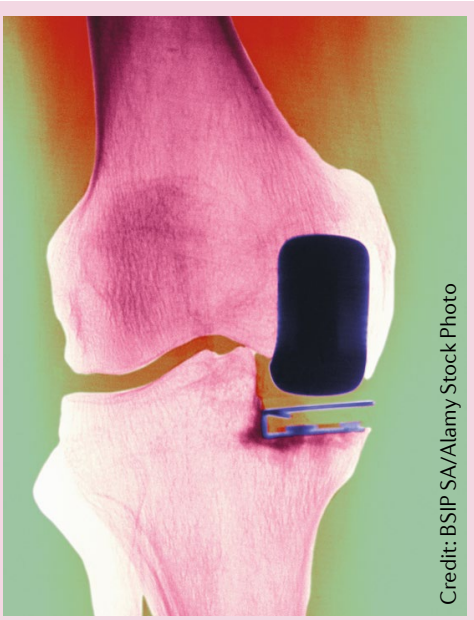

Whether the results of this trial will be enough to inform future policy regarding the choice of surgery for medial compartment knee OA remains to be seen, but the 10-year follow-up results will be eagerly awaited.

Joanna Collison

ORIGINAL ARTICLE Beard, D. J. et al. The clinical and cost-effectiveness of total versus partial knee replacement in patients with medial compartment osteoarthritis (TOPKAT): 5-year outcomes of a randomised controlled trial. Lancet https://doi.org/ 10.1016/S0140-6736(19)31281-4(2019) 